\title{
Evaluation of patients who underwent percutaneous transhepatic portal vein embolisation by Tc-99m GSA scintigraphy
}

\author{
Norifumi Kennoki ${ }^{1 \mathrm{~A}, \mathrm{~B}, \mathrm{C}, \mathrm{D}, \mathrm{E}, \mathrm{F}}$, Kiyoshi Koizumi ${ }^{2 \mathrm{E}}$, Koichi Tomita ${ }^{3 \mathrm{~B}}$, Tomohisa Moriya ${ }^{1 \mathrm{AA}, \mathrm{C}}$, Jun Otaka ${ }^{1 \mathrm{~B}, \mathrm{E}}$, \\ Kunihito Suzuki ${ }^{2 D}$, Toru Saguchi ${ }^{1 E}$, Naokazu Chiba ${ }^{3 B}$, Shigeyuki Kawachii ${ }^{3 B}$, Hiromi Serizawa ${ }^{2 B}$ \\ 'Department of Radiology, Tokyo Medical University Hospital, Tokyo, Japan \\ 2Department of Radiology, Tokyo Medical University Hachioji Medical Centre, Tokyo, Japan \\ ${ }^{3}$ Department of Digestive and Transplantation Surgery, Tokyo Medical University Hachioji Medical Centre, Tokyo, Japan
}

\section{Abstract}

Purpose: To analyse the correlation between the fold change in residual liver volume (RLV) and residual liver uptake at 15 (RLU15) before and after percutaneous transhepatic portal vein embolisation (PTPE).

Material and methods: Between August 2010 and December 2016, 20 patients who underwent PTPE were retrospectively selected. Before and three weeks after PTPE, contrast-enhanced computed tomography (CECT) and Tc-99m GSA scintigraphy were performed to analyse the fold changes in RLV and RLU15, respectively, as well as their correlation.

Results: After PTPE, a significant increase was observed in the RLV (before: $464 \pm 99 \mathrm{ml}$; after: $573 \pm 118 \mathrm{ml}, p=0.004$ ) and the RLU15 (before: $11.0 \pm 2.9 \%$; after: $17.7 \pm 3.8 \%, p=5 \times 10^{-7}$ ). The fold increase of RLV and RLU15 in all patients was $1.25 \pm 0.15$ and $1.66 \pm 0.33$, respectively. No significant correlation was observed in the fold increase in both $\operatorname{RLV}$ and RLU15 $(r=0.14, p=0.66)$. In patients no. 3 and 9 , who were outliers, the increase in RLV was minimal and RLU15 increased greatly, and these 2 patients underwent radical hepatectomy after PTPE.

Conclusions: No correlation was observed between the fold increase in RLV and RLU15 before and after PTPE. In order to accurately evaluate the residual liver function, it should be considered necessary to evaluate not only by morphological CECT volumetry, but also by functional outcome of Tc-99m GSA scintigraphy.

Residual liver volume may not necessarily reflect RLF. It may be possible to improve the radical resection rate by detecting the potential increase of RLF with RLU15 of Tc-99m GSA scintigraphy.

Key words: PTPE, Tc-99m GSA scintigraphy, LU15, future remnant liver, sinusoidal obstructive syndrome, chemotherapy-associated steatohepatitis.

\section{Introduction}

Recently, advances in medical technology have led to active and aggressive major hepatectomies. However, hepatic failure is still a fatal complication and is seen in $2.5 \%$ to $12.9 \%$ of patients [1-3]. Preoperative percutaneous transhepatic portal vein embolisation (PTPE) is known as an effective means of preventing hepatic failure by functional residual liver hypertrophy and is often performed. However, a variety of hepatic injuries, such as liver cirrhosis, as well as post-systemic chemotherapy, greatly affect the outcomes of PTPE. Although morphological computed tomography (CT) volumetry has been widely used as an easy method of evaluation before and after PTPE, it is not suitable for accurately measuring functional residual liver volume.

Correspondence address:

Dr. Norifumi Kennoki, Department of Radiology, Tokyo Medical University Hospital, 6-7-1 Nishishinjuku, Shinjuku-ku, 160-0023, Tokyo, Japan,

e-mail: norifken@yahoo.co.jp

Authors' contribution:

A Study design · B Data collection · C Statistical analysis · D Data interpretation - E Manuscript preparation · F Literature search · G Funds collection 
To date, the usefulness of Tc-99m galactosyl human serum albumin (GSA) scintigraphy for more accurate evaluation of underlying liver diseases, such as cirrhosis $[4,5]$, during both the preoperative $[2,3,6]$ and peri-PTPE period $[7,8]$, have been reported. A variety of radionuclide parameters can be used for Tc-99m GSA scintigraphy, depending on the institute. Residual liver uptake at $15 \mathrm{~min}$ utes (RLU15), which was reported by Koizumi et al., is the cumulative residual liver accumulation rate of a GSA tracer 15 minutes after its injection, and it indicates residual liver function [9]. To safely perform hepatectomies, it has been reported that the RLU15 should theoretically [10] and clinically [2] be 13 or more. A co-author of the present manuscript has already reported the usefulness of using RLU15 to predict liver failure [2]; however, to date, there has been no evaluation of the effectiveness of PTPE using RLU15. We report herein a correlation between morphological residual liver volume (RLV) and
RLU15 before and after PTPE in 20 patients, and also compare the rate of increase in RLV and RLU15 between two groups, diagnosed and evaluated as having pathological cirrhosis vs. non-cirrhosis, and post-chemotherapy vs. non-chemotherapy.

\section{Material and methods}

\section{Patient characteristics and underlying liver disease}

Patient characteristics are listed in Table 1. Between August 2010 and December 2016, 30 patients underwent preoperative PTPE. Ten of them were excluded because data on either contrast-enhanced computed tomography (CECT) or Tc-99m GSA scintigraphy were missing, and therefore 20 patients (14 men and six women, median age: 72 years, range $42-78$ years) were selected as subjects for the present study. Six patients were diagnosed as having

Table 1. Patient characteristics

\begin{tabular}{|c|c|c|c|c|c|c|c|}
\hline \multirow[t]{2}{*}{$\begin{array}{l}\text { Patient } \\
\text { no. }\end{array}$} & \multirow[t]{2}{*}{$\begin{array}{c}\text { Age } \\
\text { (years)/Sex }\end{array}$} & \multirow[t]{2}{*}{ Diagnosis } & \multirow{2}{*}{$\begin{array}{l}\text { Embolised } \\
\text { portal vein } \\
\text { branch }\end{array}$} & \multicolumn{2}{|c|}{ Underlying liver disease } & \multicolumn{2}{|c|}{$\begin{array}{l}\text { Interval to follow-up imaging } \\
\text { from PTPE procedure (days) }\end{array}$} \\
\hline & & & & $\begin{array}{l}\text { Pathological } \\
\text { cirrhosis } \\
\text { and fibrotic grade }\end{array}$ & $\begin{array}{l}\text { Chemotherapy, type } \\
\text { (no. of cycles), } \\
\text { and pathological findings }\end{array}$ & To CECT & $\begin{array}{l}\text { To Tc-99m GSA } \\
\text { scintigraphy }\end{array}$ \\
\hline 1 & $71 / \mathrm{M}$ & $\mathrm{HCC}$ & Right & No & No & 22 & 22 \\
\hline 2 & $77 / \mathrm{M}$ & MT & Right & No & $\mathrm{No}^{*}$ & 27 & 48 \\
\hline 3 & 73/M & $\mathrm{CC}$ & Right & No & No & 13 & 14 \\
\hline 4 & $61 / M$ & $\mathrm{HCC}$ & Right & Yes, 3 & No & 21 & 21 \\
\hline 5 & 74/M & $C C$ & Right & No & No & 21 & 20 \\
\hline 6 & $64 / M$ & $\mathrm{HCC}$ & Right & Yes, 4 & No & 24 & 25 \\
\hline 7 & $42 / \mathrm{F}$ & MT & Right & No & Yes, 0 (6), mild CASH & 17 & 17 \\
\hline 8 & $70 / F$ & MT & Right & No & Yes, $0+\mathrm{I}(2)$, mild SOS & 21 & 18 \\
\hline 9 & $76 / M$ & $\mathrm{HCC}$ & Right & No & No & 25 & 29 \\
\hline 10 & $49 / M$ & MT & Right & No & Yes, 0 (6), moderate CASH & 27 & 27 \\
\hline 11 & $68 / M$ & MT & Right & No & Yes, 0 (6), moderate SOS & 18 & 17 \\
\hline 12 & $72 / \mathrm{M}$ & $\mathrm{CC}$ & Right & No & No & 18 & 20 \\
\hline 13 & $72 / \mathrm{F}$ & $\mathrm{CC}$ & Right & Yes, 3 & No & 7 & 17 \\
\hline 14 & $72 / \mathrm{M}$ & $\mathrm{CC}$ & Right & No & No & 21 & 15 \\
\hline 15 & $72 / \mathrm{F}$ & $\mathrm{CC}$ & Left + Ante & - & No & 17 & 17 \\
\hline 16 & $78 / \mathrm{M}$ & $\mathrm{CC}$ & Left + Ante & No & No & 52 & 25 \\
\hline 17 & $79 / M$ & $\mathrm{HCC}$ & Left + Ante & - & No & 23 & 23 \\
\hline 18 & $75 / \mathrm{F}$ & $C C$ & Right & - & No & 25 & 25 \\
\hline 19 & $73 / \mathrm{M}$ & $\mathrm{HCC}$ & Post + P4 & No & No & 28 & 28 \\
\hline 20 & $77 / F$ & MT & Right & No & Yes, 0 (4), moderate SOS & 13 & 13 \\
\hline Median & 72 & & & & & 21.0 & 20.5 \\
\hline
\end{tabular}

HCC - hepatocellular carcinoma; CC - cholangiocarcinoma; MT - metastasis from colorectal cancer; Ante - Anterior; Post - Posterior; P4 - portal vein into segment 4; CECT - contrast-enhanced computed tomography; PTPE - percutaneous transhepatic portal vein embolization; 0 - oxaliplatin-based chemotherapy; $0+I$ - irinotecan-based chemotherapy followed by oxaliplatin-based chemotherapy; CASH - chemotherapy-associated steatohepatitis; $\mathrm{SOS}$ - sinusoidal obstructive syndrome

*Heterochronous metastasis was detected and the patient did not receive systemic chemotherapy before PTPE. The other patients with MT all had homochronous metastasis and received systemic chemotherapy before PTPE. 
hepatocellular carcinoma (HCC), six were diagnosed as having cholangiocarcinoma, and eight were diagnosed as having metastases from colorectal cancer (MT). Five out of six MT patients underwent systemic chemotherapy before PTPE.

\section{Interventional radiology devices and embolic agents}

A 6-Fr long sheath and a J-curved and loop-shaped 5.5-Fr balloon catheter (Selecon MP catheter II, Terumo, Tokyo, Japan) were used in every procedure (Figure 1). Absolute ethanol (Absolute Ethanol ${ }^{\mathrm{TM}}$, FUSO Pharmaceutical Industries, Ltd., Osaka, Japan) was used as an embolic material in all cases and was also used for the ethanol-lipiodol mixture, which contained $2 \mathrm{ml}$ of ethiodised oil (Lipiodol $^{\mathrm{TM}}$, Guerbet Japan, Tokyo, Japan) and $8 \mathrm{ml}$ of ethanol. The maximum dose of ethanol was decided to be less than $0.2 \mathrm{ml} / \mathrm{kg}$ of body weight, which was considered a safe dose. Gelatine sponges (GS, Serescue ${ }^{\mathrm{TM}}$ Nippon Kayaku Co., Ltd., Tokyo, Japan) were used for approach tract embolisation.

\section{PTPE procedure}

A routine method by which we perform embolisation of the portal vein branch of the right hepatic lobe is described below, which is similar to the PTPE outline using absolute ethanol reported by Satake et al. [11]. In all cases, the percutaneous transhepatic and ipsilateral approach was used. The portal vein of segment no. 5 (P5) or P6 was usually punctured with a $21-\mathrm{G}$ needle under ultrasound guidance. A 6-Fr long sheath and a 5.5-Fr loop-shaped balloon catheter was coaxially inserted and advanced to the main trunk of the portal vein. Digital subtraction angiography (DSA) was performed, and whole portal vein images were confirmed (Figure 2A). A loop was made at the tip of the balloon catheter within the splenic vein. The tip of the catheter was inserted into the anterior branch of the portal vein and the balloon was inflated at the beginning of the anterior branch. Selective DSA was performed from this point (Figure 2B), which was also a test injection. The ethanol and lipiodol mixture was carefully and slowly injected to avoid backflow, particularly back into the left portal branch (Figure 2C). The dose of the embolic material was set at approximately the same as that of the test injection. Next, a 10-minute wait was necessary, until the endothelium of the portal vein became damaged and an embolic effect appeared. The balloon catheter was removed, DSA was performed from the sheath, and we confirmed that the embolised anterior branch of the portal vein was not displayed on the image. Next, the other J-curved balloon catheter was inserted into the portal vein trunk, and the tip of the balloon was placed on the beginning of the posterior branch as distally as possible because this makes portal vein reconstruction easier for the surgeon during hepatectomy. Selective DSA (test injection)

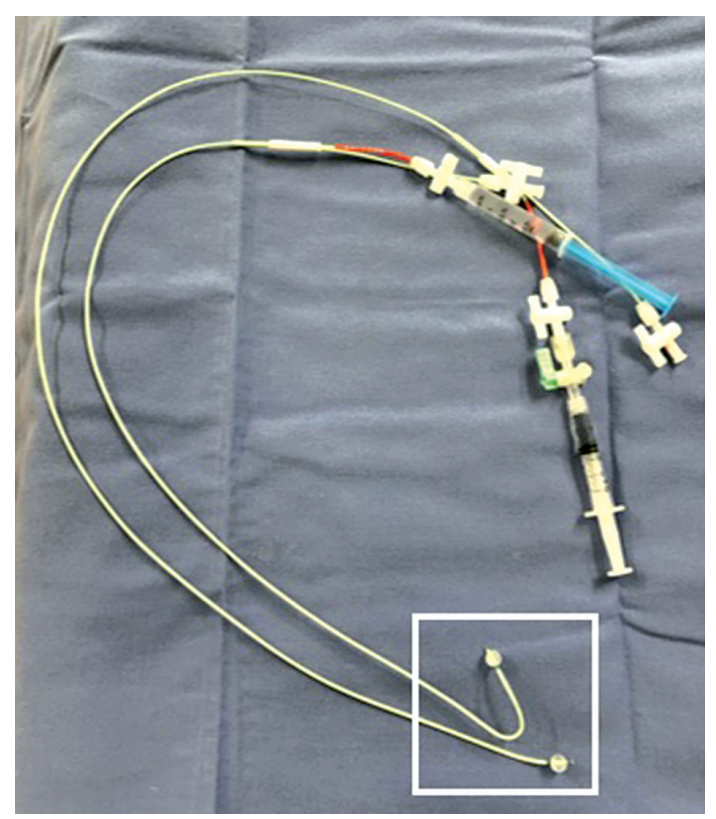

Figure 1. Photographs of the balloon catheters that were used. The size of the catheters was $5.5 \mathrm{Fr}$, and 2 types of tips, namely J-shaped and loopshaped, were used

was performed during balloon occlusion (Figure 2D). The ethanol-lipiodol mixture was injected from the sheath at the same dose as the test injection (Figure 2E). Finally, access tract embolisation was necessary to prevent bleeding from the puncture site (Figure 3). A GS was cut into equal-sized thin strips, which were rounded into a cylindrical shape (Figure 3C). A second sheath was prepared and cut at approximately $5 \mathrm{~cm}$ intervals (Figure 3D), and the cylindrical shaped GS was put into the cylindrical cut sheath. The cut sheath within the GS was inserted from behind of the first sheath, and the GS was pushed into the tract by the inner cylinder with a guidewire from behind the first sheath, and the tract was packed with GS in the form of a mould (Figure 3E).

\section{Parameters and evaluation items}

Before and approximately three weeks after PTPE, CECT and Tc-99m GSA scintigraphy were performed. The RLV and the RLU15 were used as the main parameter. CT volumetry, including RLV, was calculated by Synapse Vincent (Fujifilm Holdings Corporation, Tokyo, Japan). Liver uptake at 15 minutes (LU15) is the ratio of cumulative liver uptake from 15 to 16 minutes after GSA administration divided by the total GSA dose, and its formula is as below [9].

$$
\mathrm{LU} 15=\frac{\int_{15}^{16} \mathrm{C}(\mathrm{t}) \mathrm{dt}}{\text { total injected dose }} \times 100(\%)
$$

$\mathrm{C}(\mathrm{t})$ is the time-activity curves in which the region of interest is set in the liver in a continuous image taken from the anterior surface. RLU15 is the value obtained by multiplying LU15 by the residual count ratio calculated from single-photon emission computed tomography. 

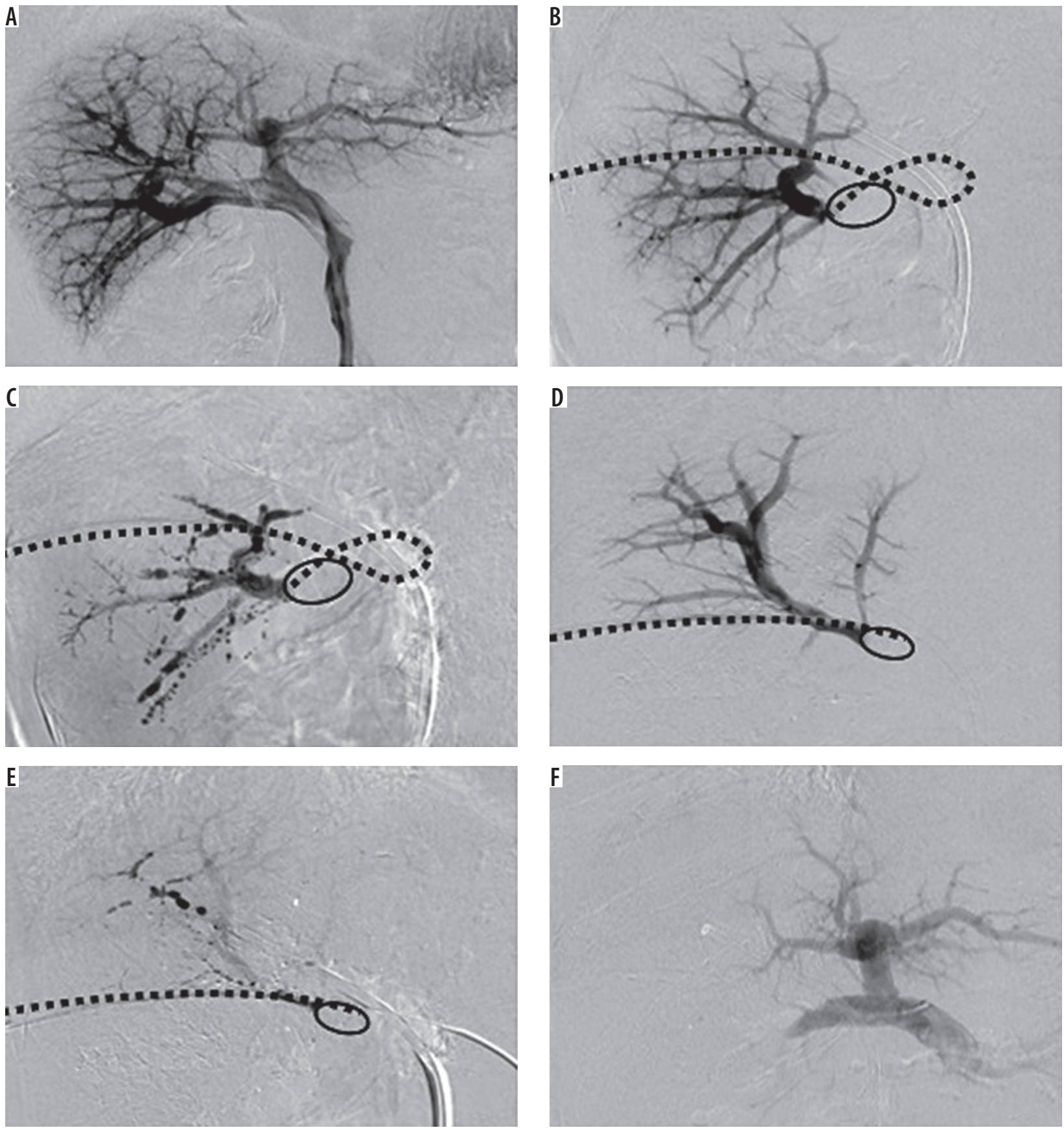

Figure 2. Digital subtraction angiography (DSA) during the percutaneous transhepatic portal vein embolisation (PTPE) procedure. A) The tip of the sheath was placed on the trunk of the portal vein, and contrast material was injected into the liver. The entire portal vein trunk and branches were displayed. B) A loop-shaped balloon catheter was turned back at the splenic vein and then its tip was inserted into the anterior segment branch, and after balloon occlusion, DSA of the anterior segment branch was performed. C) The ethanol-lipiodol mixture was carefully injected into the anterior segment branch while confirming the absence of reflux. D) A J-shaped balloon catheter was inserted into the beginning of the posterior branch, the balloon was occluded, and the contrast material was injected from the sheath. E) The ethanol-lipiodol mixture was carefully injected into the posterior segment branch while confirming the absence of reflux. F) In the final image, the portal vein of the right lobe was completely embolised, and only the portal vein of the left lobe was visualised

When evaluating residual liver function before hepatectomy, the desirable RLU15 value is reported to be more than 13 [10].

As the primary endpoint, RLV, RLU15, the fold change in RLV ( $\triangle \mathrm{RLV})$, the fold change in RLU15 ( $\triangle$ RLU15), and correlation between $\triangle R L V$ and $\triangle$ RLU15 were obtained. $\triangle \mathrm{RLV}$ and $\triangle \mathrm{RLU} 15$ were calculated as follows:

$$
\Delta \mathrm{RLV}=\frac{\text { after RLV }}{\text { before RLV }}, \Delta \mathrm{RLU} 15=\frac{\text { after RLU15 }}{\text { before RLU15 }}
$$

The samples of 16 patients who underwent radical hepatectomy were histopathologically evaluated. The liver cirrhosis group was defined as cases of fibrosis grade $(\mathrm{F})$ 3 or F4 pathologically diagnosed according to the classification reported by Desmet et al. [12].

Sinusoidal obstructive syndrome (SOS) and chemotherapy-associated steatohepatitis (CASH) are wellknown hepatic impairments caused by systemic chemotherapy for colorectal cancer, and they were evaluated 

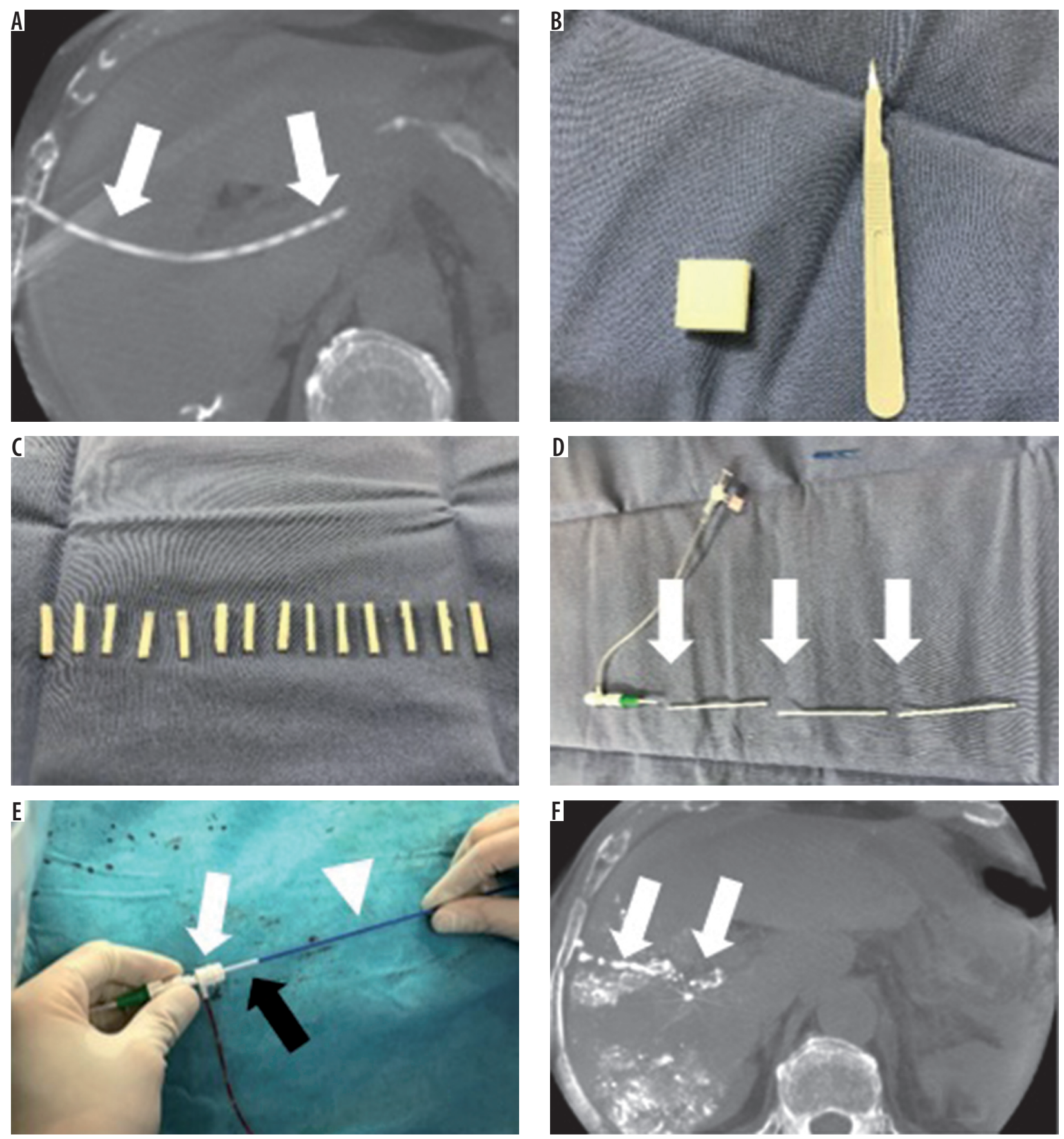

Figure 3. Photographs demonstrating tract embolisation. A) The percutaneous transhepatic tract (arrows) is embolised to prevent bleeding from the puncture site. B) A gelatine sponge (GS) and a sharp disposable pointed scalpel. C) The GS was cut into 2-mm rectangular strips and manually rounded into a cylindrical shape. D) A new sheath was divided into three equal parts of about $5 \mathrm{~cm}$ (arrows), and the cut GS was put into the cut sheath. The GS was soaked with a small amount of contrast agent. E) This cut sheath (black arrow) was inserted into the first sheath (white arrow) and pushed with the inner tube (white arrowhead) under fluoroscopy. F) The tract was embolised completely in the shape of the mould (arrows)

based on the scores reported by Rubbia-Brandt et al. [13]. $\triangle \mathrm{RLV}$ and $\Delta \mathrm{RLU} 15$ were compared between the two groups, i.e. the cirrhosis group $(n=3)$ vs. the non-cirrhosis group $(n=13)$, and the chemotherapy group $(n=5)$ vs. the non-chemotherapy group $(n=15)$.

As a secondary endpoint, $\%$ future remnant liver (FRL) change rates (increase $\% \mathrm{FRL}$ ) were calculated using the following formulas:

$\% \mathrm{FRL}=\frac{\mathrm{FRLV}}{\mathrm{TLV}-\mathrm{TV}} \times 100 \%, \Delta \% \mathrm{FRL}=\frac{\text { after } \% \mathrm{FRL}-\text { before } \% \mathrm{FRL}}{\text { before } \% \mathrm{FRL}}$
(FRLV - future remnant liver volume; TLV - total liver volume; TV - tumour volume)

Adverse events were evaluated using CTCAE v4.0 software.

\section{Statistical analysis}

Ratios were expressed as means and standard deviations. For the differences in increases in RLV and RLU15 before and after PTPE, Student's $t$-test was used. For two-group 
comparisons, Wilcoxon's rank sum test was used. A p-value less than 0.05 was considered to indicate a significant difference between two groups.

\section{Results}

The median amount of ethanol used was 7.5 (3.67-10.0) $\mathrm{ml}$. The embolic areas were the right lobe in 16 patients and the three segments in four patients. Of these four patients three were embolised in the left lobe and anterior segment, and one was embolised in the right lobe and segment 4 . The median intervals from PTPE to CECT, to Tc-99m GSA scintigraphy, and to surgery were 21 (7-52) days, 21 (13-48) days, and 31 (20-56) days, respectively. Radical resection was performed in 16 patients (curative hepatectomy rate: $80 \%$ ), and these patients were pathologically evaluated. In the other four patients (nos. 15, 17, 18 , and 19), hepatectomy was not performed because the progression of the original disease was seen (Table 2).

\section{Primary endpoint}

Total liver volume before and after PTPE were 1304 \pm 278 $\mathrm{ml}$ and $1249 \pm 254 \mathrm{ml}$, respectively, and no significant difference was seen $(p=0.53)$. A significant increase was observed in the RLV $(464 \pm 99 \mathrm{ml}$ and $573 \pm 118 \mathrm{ml}$, $p=0.004$, Figure 4 ) before and after PTPE. $\Delta$ RLV in all cases was $1.25 \pm 0.15$. Total liver LU15 before and after PTPE were $28.8 \pm 4.9 \%$ and $27.3 \pm 4.9 \%$, respectively, which indicated no significant difference $(p=0.35)$. The RLU 15 before and after PTPE were $11.0 \pm 2.9 \%$ and $17.7 \pm 3.8 \%$, respectively, with a significant increase $\left(p=5 \times 10^{-7}\right.$, Figure 4$) . \Delta$ RLU15 in all cases was $1.66 \pm 0.33$. No significant correlation was observed in either RLV or RLU15 before and after PTPE $(r=0.14, p=0.66$, Figure $5 \mathrm{~A}$ ), although both RLV and RLU15 were significantly increased. Outliers were patients nos. 3, 9, and 16, and after these patients were excluded, the correlation coefficient increased to $0.76(p=0.005$, Figure $5 B)$. The results

Table 2. Morphological and functional parameters obtained from PTPE, PTPE complications, and follow-up

\begin{tabular}{|c|c|c|c|c|c|c|c|c|c|}
\hline \multirow{2}{*}{$\begin{array}{l}\text { Patient } \\
\text { no. }\end{array}$} & \multicolumn{3}{|c|}{ RLV (ml) } & \multicolumn{3}{|c|}{ RLU15 (\%) } & \multirow[t]{2}{*}{ Complications } & \multirow{2}{*}{$\begin{array}{c}\text { Radical } \\
\text { resection* }\end{array}$} & \multirow{2}{*}{$\begin{array}{c}\text { Reason that radical } \\
\text { resection } \\
\text { could not be performed }\end{array}$} \\
\hline & $\begin{array}{l}\text { Before } \\
\text { PTPE }\end{array}$ & $\begin{array}{l}\text { After } \\
\text { PTPE }\end{array}$ & $\Delta \mathrm{RLV}$ & $\begin{array}{l}\text { Before } \\
\text { PTPE }\end{array}$ & $\begin{array}{l}\text { After } \\
\text { PTPE }\end{array}$ & $\Delta$ RLU15 & & & \\
\hline 1 & 418 & 516 & 1.23 & 8.5 & 14.2 & 1.67 & - & Yes & - \\
\hline 2 & 501 & 598 & 1.19 & 9.8 & 17.6 & 1.80 & - & Yes & - \\
\hline 3 & 378 & 360 & 0.95 & 5.9 & 11.4 & 1.93 & - & Yes & - \\
\hline 4 & 541 & 693 & 1.28 & 11.0 & 19.4 & 1.76 & - & Yes & - \\
\hline 5 & 459 & 562 & 1.22 & 4.3 & 7.6 & 1.77 & - & Yes & - \\
\hline 6 & 481 & 675 & 1.40 & 10.6 & 16.8 & 1.58 & - & Yes & - \\
\hline 7 & 519 & 625 & 1.20 & 16.5 & 22.9 & 1.39 & - & Yes & - \\
\hline 8 & 313 & 470 & 1.50 & 7.5 & 18.0 & 2.40 & - & Yes & - \\
\hline 9 & 553 & 633 & 1.14 & 10.3 & 22.9 & 2.22 & - & Yes & - \\
\hline 10 & 558 & 748 & 1.36 & 10.9 & 17.2 & 1.58 & $\begin{array}{l}\text { ALT and AST } \\
\text { elevation } \\
\text { (grade 4) }\end{array}$ & Yes & - \\
\hline 11 & 612 & 637 & 1.04 & 13.6 & 17.3 & 1.27 & - & Yes & - \\
\hline 12 & 687 & 845 & 1.23 & 10.3 & 18.6 & 1.61 & - & Yes & - \\
\hline 13 & 495 & 616 & 1.24 & 11.0 & 22.0 & 1.69 & - & Yes & - \\
\hline 14 & 445 & 609 & 1.37 & 12.2 & 22.0 & 1.80 & - & No & Multiple dissemination \\
\hline 15 & 406 & 484 & 1.19 & 13.0 & 16.7 & 1.28 & - & Yes & - \\
\hline 16 & 391 & 592 & 1.51 & 14.7 & 15.0 & 1.02 & $\begin{array}{l}\text { Cholangitis } \\
\text { (grade 3) }\end{array}$ & No & Tumour progression \\
\hline 17 & 509 & 492 & 0.97 & 12.7 & 15.2 & 1.20 & - & No & Tumour progression \\
\hline 18 & 358 & 456 & 1.27 & 14.8 & 21.9 & 1.48 & - & No & Tumour progression \\
\hline 19 & 297 & 411 & 1.38 & 10.1 & 18.8 & 1.86 & - & Yes & - \\
\hline 20 & 348 & 437 & 1.26 & 11.9 & 23.9 & 1.93 & - & Yes & - \\
\hline Mean & $464 \pm 99$ & $573 \pm 118$ & $1.25 \pm 0.15$ & $11.0 \pm 2.9$ & $17.7 \pm 3.8$ & $1.66 \pm 0.33$ & & & \\
\hline
\end{tabular}

PTPE - percutaneous transhepatic portal vein embolisation; RLV - residual liver volume; RLU15 - residual liver uptake at 15 minutes 

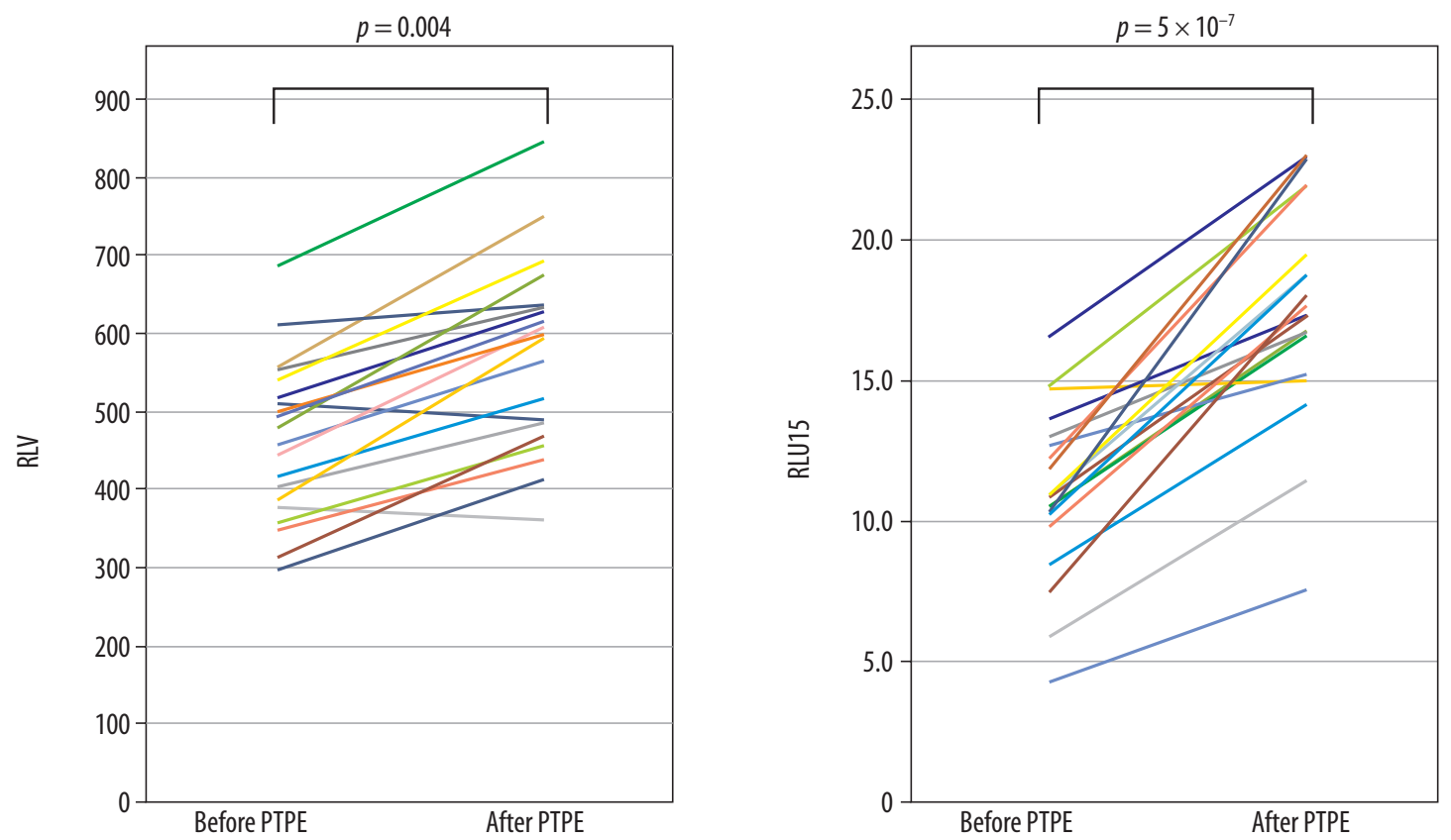

Figure 4. Changes in residual liver volume (RLV) and residual liver uptake at 15 minutes (RLU15) before and after PTPE. The RLV (before: $464 \pm 99 \mathrm{ml}$; after: $573 \pm 118 \mathrm{ml}, p=0.004$ ) and RLU 15 (before: $11.0 \pm 2.9 \%$; after: $17.7 \pm 3.8 \%, p=5 \times 10^{-7}$ ) were significantly increased after PTPE
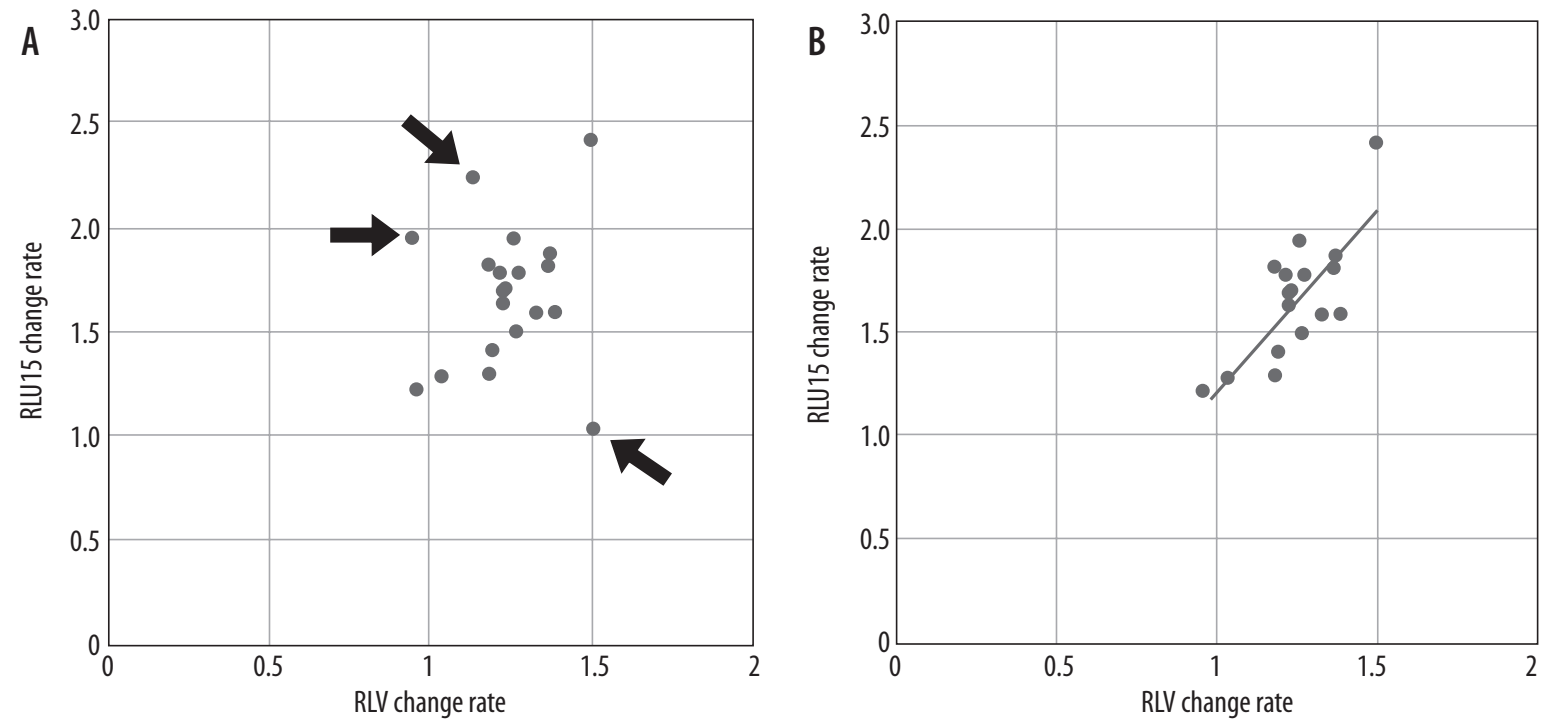

Figure 5. Correlation between rates of changes in RLV and RLU15. A) When all patients were analysed, no correlation was observed $(r=0.14, p=0.66)$. The three outliers were patients nos. 3, 9, and 16 (arrows). B) When the three outliers were excluded, a positive correlation was obtained $(r=0.76, p<0.005)$

Table 3. Outliers of the RLV and RLU15 results

\begin{tabular}{|l|c|c|c|c|}
\hline \multirow{2}{*}{ Outliers of the RLV results } & Patient no. & $\Delta$ RLV & $\Delta$ RLU15 & Outcomes \\
\cline { 2 - 4 } & 3 & 0.95 & 1.93 & Radical hepatectomy was performed \\
\hline An outlier of the RLU15 results & 9 & 1.14 & 2.22 & \\
& 16 & 1.51 & 1.02 & $\begin{array}{c}\text { Progression of the present disease was confirmed during surgery } \\
\text { and hepatectomy could not be performed }\end{array}$ \\
\hline
\end{tabular}

of these three outliers are shown in Table 3. In patients nos. 3 and 9 there was a minimal increase in RLV and a large increase in RLU15, and these two patients were able to undergo radical hepatectomy after PTPE. Patient no. 16 showed a large increase in RLV and a small increase in RLU15, and radical hepatectomy could not be completed because of tumour progression. The outcomes of the two-group comparisons are listed in Table 4. Pathological cirrhosis was observed in patients nos. 5, 7, and 14 (Figure 6A), and the other 13 patients, including those with 
Table 4. Outcomes of patients with cirrhotic liver vs. non-cirrhotic liver and patients undergoing systemic chemotherapy vs. no systemic chemotherapy before PTPE

\begin{tabular}{|l|c|c|c|c|}
\hline & Cirrhotic (3) vs. non-cirrhotic (13) & $p$-value & Chemo (5) vs. no chemo (15) & $p$-value \\
\hline$\Delta R L V$ & 1.28 vs. 1.22 & 0.12 & 1.26 vs. 1.23 & 0.76 \\
\hline$\Delta R L U 15$ & 1.69 vs. 1.77 & 0.59 & 1.58 vs. 1.69 & 0.97 \\
\hline
\end{tabular}
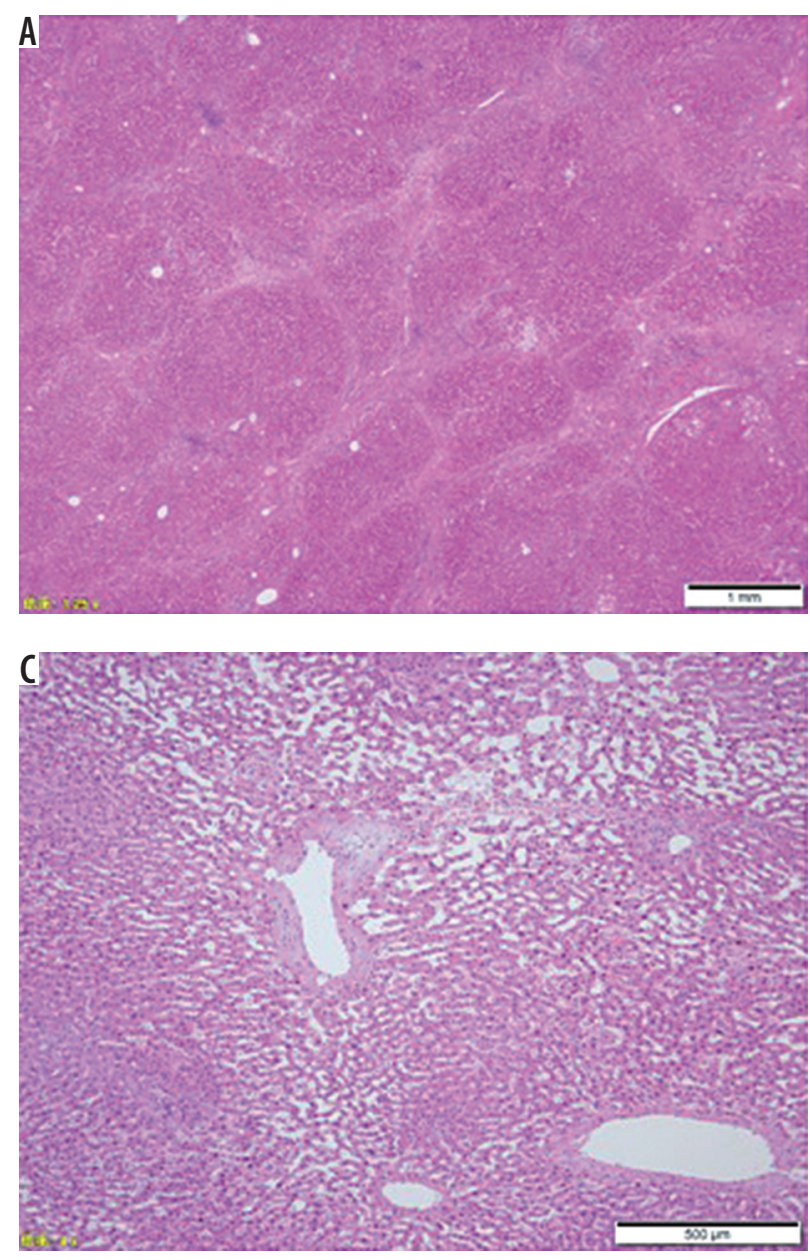

HCC, had no obvious fibrosis. There were no significant differences between cirrhotic and non-cirrhotic groups regarding both $\triangle \mathrm{RLV}(1.28$ vs. $1.22, p=0.12)$ and $\Delta \mathrm{RLU} 15$ (1.69 vs. $1.77, p=0.59$ ). Five patients (nos. $7,8,10,11$, and 20) underwent two to six courses of systemic chemotherapy before PTPE. Oxaliplatin-based chemotherapy was performed in these five patients. Only one patient (no. 8) switched to irinotecan-based chemotherapy because of an allergy to oxaliplatin (Table 1). No significant differences were observed between chemotherapy and non-chemotherapy groups in both $\Delta \mathrm{RLV}(1.26$ vs. $1.23, p=0.76)$ and $\triangle$ RLU 15 ( 1.58 vs. $1.69, p=0.97$ ).

\section{Secondary endpoint}

The $\%$ FRL increase was $34.4 \pm 25.2 \%$. At the time of injection of ethanol, local abdominal pain was recognised in all patients; however, it was under self-control by the

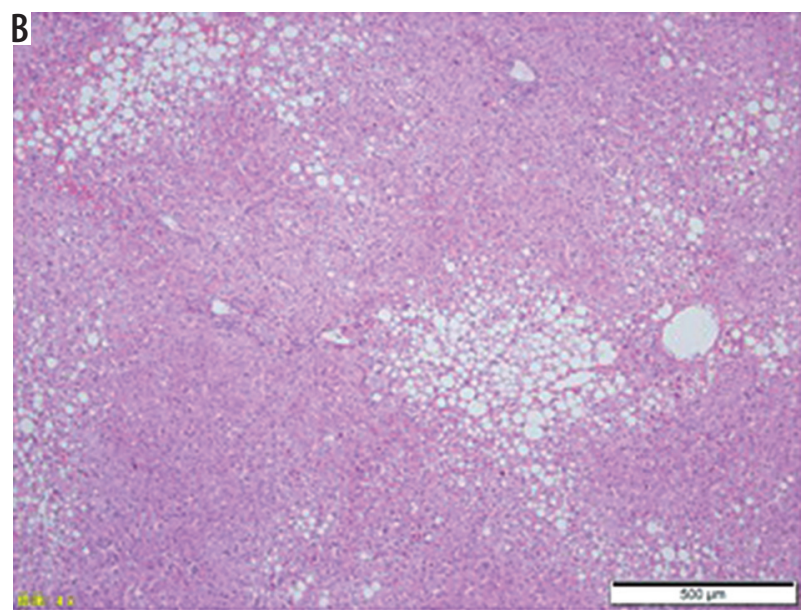

Figure 6. Photographs of liver pathology of three representative patients. All samples were stained with haematoxylin and eosin; original magnification: $\times 40$. The sample in $(A)$ was prepared for the evaluation of fibrotic grade, and $(B)$ and $(C)$ were prepared to evaluate post-chemotherapeutic effects. A) Patient no. 6 was evaluated as fibrosis grade 4. B) Patient no. 10 underwent six courses of FOLFOX with Bevacizumab and was concluded to have moderate CASH (stenosis in 30\% of hepatocytes). C) Patient no. 20 received four courses of FOLFOX with Bevacizumab and was concluded to have moderate SOS (centrilobular involvement of two-thirds of the lobular surface)

administration of analgesics. Major adverse events are listed in Table 2. Grade 3 cholangitis, which requires endoscopic retrograde biliary drainage (ERBD), was found in one patient. Grade 4 alanine aminotransferase (ALT) and aspartate transaminase (AST) elevation were observed in one patient, grade 3 ALT elevation in 10 patients, grade 3 AST elevation in seven patients, grade 3 bilirubin elevation in one patient, and grade 3 thrombocytopenia in one patient; however, these symptoms were adequately treated by conservative treatments. Regarding postembolic syndromes, grade 1 fever was observed in five patients, grade 2 nausea in five patients, and grade 1 vomiting in one patient.

\section{Discussion}

Some studies have compared morphological RLV with residual liver function (RLF), which is another radionu- 
clide parameter that is different from RLU15 obtained from Tc-99m GSA scintigraphy, before and after PTPE $[7,8,14]$. The fold change in RLV and RLF were 1.28 to 1.32 and 1.63 to 1.7 , respectively, and generally, the increase in the rate of change in RLF is known to be much greater than that of $\operatorname{RLV}[3,6,8,15]$. In the present study, the fold changes in RLV and RLU15 were $1.25 \pm 0.15$ and $1.66 \pm 0.33$, respectively, which is equivalent to those of previous reports. No correlation was seen between the fold change in RLV and RLU15 in all patients. Kono et al. reported that no correlation was observed between morphological RLV and residual liver R max (a radionuclide parameter) before and after PTPE in all patients $(r=0.105$, $p=0.803$ ) [8]. A good correlation between these parameters was observed when the patients were narrowed down to the good response group that showed a large increase in $\mathrm{R} \max (r=0.826, p=0.037)$. Nanashima et al. found a good correlation between CT volumetry before PTPE and RI volumetry $(r=0.69)$; however, a very weak correlation was found between CT volumetry and RI volumetry after PTPE $(r=0.03)$ [15]. From these previous reports, it is suggested that although PTPE increases both RLV and RLF, these parameters may not be simply well correlated. Therefore, it is clinically important and desirable to analyse both parameters for more accurate evaluation of liver function. We divided the outliers into two types. Type A, comprising two of the three outliers, showed a small increase in RLV but a large increase in RLU15 (Table 3A). Type B, comprising one of the three outliers, showed a large increase in RLV but a small increase in RLU15 (Table 3B). Compared with morphological RLV, RLF values obtained from GSA scintigraphy are known to be better correlated with postoperative albumin level [15], prothrombin time [10], total bilirubin $[10,15]$, preoperative indocyanine green retention rate at $15 \mathrm{~min}$ (ICGR 15) $[9,10]$, and postoperative indocyanine green $[6,15]$. Therefore, in patients of type $A$, even if only a small increase in RLV is observed, a large increase in RLU15 is likely to indicate sufficient regeneration of the residual liver. As a result, we were able to perform surgery on all type A patients. On the other hand, in type B, two hypotheses were clinically inferred. The first hypothesis was that the underlying liver disease was much worse, and liver regeneration might take a comparatively long time. The remnant liver is known to continue to undergo hypertrophy after PTPE and postoperatively $[3,15]$. The second hypothesis was that there was rapid progression of the present liver disease and that regeneration itself was poor. In the case of patient no. 16, the latter hypothesis was thought to be true because progression of the present disease was found during surgery and hence curative operation was not performed. Many studies have reported that sufficient RLV and RLF increases in patients with liver cirrhosis may not be observed after PTPE [3,16-19]. In the present study, the fold change in RLV appeared to be good (1.28), but the RLU15 remained at 1.69. In the case of cirrhotic patients,
Kono et al. speculated that an increase in RLV reflects not an increase in the number of functional hepatocytes but instead an increase in the interstitial area [8]. This hypothesis suggests that in patients with liver cirrhosis, even if a good increase in RLV is observed, careful evaluation is required. To our knowledge, there are few studies comparing RLF in post-chemotherapy and non-chemotherapy groups before and after PTPE. Patients undergoing systemic chemotherapy after PTPE did not show a significant decrease in the rate of increase in RLV compared with the non-chemotherapy group $[17,20,21]$. On the other hand, de Baere et al. found that the rate of increase in RLV significantly decreased in the patient group, using the platinum formulation [22]. Narita et al. reported that the rate of increase in RLV significantly decreased in the SOS group $(16.8 \pm 24.0$ vs. $55.6 \pm 32.5, p<0.001)$ [23]. The median number of chemotherapy cycles was 10 (range, 2-44), and the grades of SOS were mild in five patients, moderate in four patients, and severe in two patients. Ninety per cent of the patients with SOS received more than six cycles of chemotherapy and/or underwent oxaliplatin-based chemotherapy. In the present study, the chemotherapy group had equivalent outcomes in both RLV and RLU15 compared with the non-chemotherapy group, and consequently, curative hepatectomy was performed. Five cases in the chemotherapy group were pathologically evaluated, as follows: Moderate SOS was found in patients nos. 11 and 20, mild SOS in patient no. 8, moderate CASH was seen in patient no. 10, and mild CASH was seen in patient no. 7. No severe cases of both SOS and CASH were observed. An insufficient increase in RLU15 was observed only in patient no. 11 . Thus, for up to six cycles of chemotherapy, it seems that systemic chemotherapy before PTPE might not affect increases in RLV and RLU15.

According to a systematic review of PTPE, \% FRL was used for calculating the hypertrophy response of residual liver in $68.2 \%$ of reported studies [17]. The average and width of $\%$ FRL volume increase were used in $37.9 \pm 0.1 \%$ and $20.5 \%$ to $69.4 \%$, respectively. Sakuhara et al. reported that, in a study of 143 patients who underwent PTPE using ethanol, the \% FRL volume increase and resection rate were $33.6 \%$ and $84 \%$, respectively [24]. In the present study, the \% FRL volume increase and resection rate were $34.4 \pm 25.2 \%$ and $80 \%$, respectively, which were similar to the previous study. The rate of PTPE complications was reported as $2.2 \%$ to $15 \%$ [25-27]. As a mild complication that is specific to the use of ethanol, pain at the time of injection may be reported. However, in our present study, most cases of pain were relieved within a few minutes with or without analgesics, similarly to a previous publication [24]. Pneumothorax, cholangitis, PVT, etc. were reported as major complications [25-27]. Rare but fatal cases of pulmonary embolism and cholangitis after PTPE have been reported [28]. In the present study, patient no. 16 had cholangitis on day 12 after PTPE, and although ERBD had been performed 14 days before PTPE, it was 
performed again. Radical hepatectomy was attempted after two months; however, the tumour was unresectable due to tumour progression. A serious complication after PTPE was only observed in this patient.

\section{Limitations}

This study has several limitations. Firstly, it is a retrospective study with only a few patients. All pathological specimens were obtained after surgery, and therefore they were affected by both PTPE and surgery. As hypertrophy of the residual liver continues even after hepatectomy, the final postoperative residual liver function was not evaluated. The outcomes after hepatectomy appear to most greatly affect the patients' prognosis; however, the success rate of the operation depends not only on residual liver function but also on operation time, amount of bleeding, and whether or not vascular reconstruction was performed. In the present study, the effect of pure PTPE was evaluated.

\section{Conclusions}

No correlation was found between rates of changes in RLV and RLU15 both before and after PTPE. Outliers in which RLU15 increased despite a small increase in RLV suggested that these may be cases in which to consider surgery. Severe liver injury did not occur upon systemic chemotherapy for up to six cycles, and the effects of PTPE were similar to those in patients with liver cancer, who did not receive chemotherapy.

\section{Acknowledgments}

The authors thank Dr. Mitsuru Okubo and Mr. Kohei Fujimura for their excellent help in data collection and analysis.

\section{Conflict of interest}

The authors report no conflict of interest.

\section{References}

1. Shindoh J, Tzeng CW, Aloia TA, et al. Safety and efficacy of portal vein embolization before planned major or extended hepatectomy: an institutional experience of 358 patients. J Gastrointest Surg 2014; 18: 45-51.

2. Chiba N, Shimazu M, Takano K, et al. Predicting hepatic failure with a new diagnostic technique by preoperative liver scintigraphy and computed tomography: a pilot study in 123 patients undergoing liver resection. Patient Saf Surg 2017; 11: 29.

3. Yumoto Y, Yagi T, Sato S, et al. Preoperative estimation of remnant hepatic function using fusion images obtained by $99 \mathrm{mTc}$-labelled galactosyl-human serum albumin liver scintigraphy and computed tomography. Br J Surg 2010; 97: 934-944.

4. Kokudo N, Vera DR, Koizumi M, et al. Recovery of hepatic asialoglycoprotein receptors after major hepatic resection. J Nucl Med 1999; 40: 137-141.

5. Wu J, Ishikawa $\mathrm{N}$, Takeda $\mathrm{T}$, et al. The functional hepatic volume assessed by $99 \mathrm{~m}$ Tc-GSA hepatic scintigraphy. Ann Nucl Med 1995; 9: 229-235.

6. Mitsumori A, Nagaya I, Kimoto S, et al. Preoperative evaluation of hepatic functional reserve following hepatectomy by technetium-99m galactosyl human serum albumin liver scintigraphy and computed tomography. Eur J Nucl Med 1998; 25: 1377-1382.

7. Kasai Y, Hatano E, Iguchi K, et al. Prediction of the remnant liver hypertrophy ratio after preoperative portal vein embolization. Eur Surg Res 2013; 51: 129-137.

8. Kono Y, Kariya S, Komemushi A, et al. Comparison of Tc-99m GSA scintigraphy and CT volumetry for evaluation in portal vein embolization. Minim Invasive Ther Allied Technol 2014; 23: 241-246.

9. Koizumi K, Uchiyama G, Arai T, et al. A new liver functional study using Tc-99m DTPA-galactosyl human serum albumin: evaluation of the validity of several functional parameters. Ann Nucl Med 1992; 6: 83-87.
10. Uetake M, Koizumi K, Yagawa A, et al. Use of Tc-99m DTPA galactosyl human serum albumin to predict postoperative residual liver function. Clin Nucl Med 1999; 24: 428-434.

11. Satake M, Hayashi T, Iwata R, et al. Percutaneous transhepatic portal vein embolization using absolute ethanol. Nichidoku-Iho 2007; 52: 94-99. (Japanese)

12. Desmet VJ, Gerber M, Hoofnagle JH, et al. Classification of chronic hepatitis: diagnosis, grading and staging. Hepatology 1994; 19: 1513-1520.

13. Rubbia-Brandt L, Audard V, Sartoretti P, et al. Severe hepatic sinusoidal obstruction associated with oxaliplatin-based chemotherapy in patients with metastatic colorectal cancer. Ann Oncol 2004; 15: 460-466.

14. Nishiyama Y, Yamamoto Y, Hino I, et al. 99mTc galactosyl human serum albumin liver dynamic SPET for pre-operative assessment of hepatectomy in relation to percutaneous transhepatic portal embolization. Nucl Med Commun 2003; 24: 809-817.

15. Nanashima A, Tobinaga $S$, Abo T, et al. Relationship of hepatic functional parameters with changes of functional liver volume using technetium-99m galactosyl serum albumin scintigraphy in patients undergoing preoperative portal vein embolization: a follow-up report. J Surg Res 2010; 164: e235-e242.

16. Farges O, Belghiti J, Kianmanesh R, et al. Portal vein embolization before right hepatectomy: prospective clinical trial. Ann Surg 2003; 237: 208-217.

17. Van Lienden KP, Van den Esschert JW, De Graaf W, et al. Portal vein embolization before liver resection: a systematic review. Cardiovasc Intervent Radiol 2013; 36: 25-34.

18. Cotroneo AR, Innocenti P, Marano G, et al. Pre-hepatectomy portal vein embolization: single center experience. Eur J Surg Oncol 2009; 35: 71-78. 
19. Ko GY, Sung KB, Yoon HK, et al. Preoperative portal vein embolization with a new liquid embolic agent. Radiology 2003; 227: 407-413.

20. Zorzi D, Chun YS, Madoff DC, et al. Chemotherapy with bevacizumab does not affect liver regeneration after portal vein embolization in the treatment of colorectal liver metastases. Ann Surg Oncol 2008; 15: 2765-2772.

21. Covey AM, Brown KT, Jarnagin WR, et al. Combined portal vein embolization and neoadjuvant chemotherapy as a treatment strategy for resectable hepatic colorectal metastases. Ann Surg 2008; 247: 451-455.

22. de Baere T, Teriitehau C, Deschamps F, et al. Predictive factors for hypertrophy of the future remnant liver after selective portal vein embolization. Ann Surg Oncol 2010; 17: 2081-2089.

23. Narita M, Oussoultzoglou E, Chenard MP, et al. Sinusoidal obstruction syndrome compromises liver regeneration in patients undergoing two-stage hepatectomy with portal vein embolization. Surg Today 2011; 41: 7-17.
24. Sakuhara Y, Abo D, Hasegawa Y, et al. Preoperative percutaneous transhepatic portal vein embolization with ethanol injection. AJR Am J Roentgenol 2012; 198: 914-922.

25. Abulkhir A, Limongelli P, Healey AJ, et al. Preoperative portal vein embolization for major liver resection: a meta-analysis. Ann Surg 2008; 247: 49-57.

26. Di Stefano DR, de Baere T, Denys A, et al. Preoperative percutaneous portal vein embolization: evaluation of adverse events in 188 patients. Radiology 2005; 234: 625-630.

27. Kodama Y, Shimizu T, Endo H, et al. Complications of percutaneous transhepatic portal vein embolization. J Vasc Interv Radiol 2002; 13: $1233-1237$.

28. Giraudo G, Greget M, Oussoultzoglou E, et al. Preoperative contralateral portal vein embolization before major hepatic resection is a safe and efficient procedure: a large single institution experience. Surgery 2008; 143: 476-482. 